\title{
Scalar-Flat Lorentzian Einstein-Weyl Spaces
}

\author{
David M.J.Calderbank \\ Department of Mathematics and Statistics, \\ University of Edinburgh, \\ King's Buildings, Mayfield Road, Edinburgh EH9 3JZ, Scotland UK \\ Maciej Dunajski \\ Mathematical Institute, \\ University of Oxford, 24-29 St Giles, Oxford OX1 3LB, UK.
}

\begin{abstract}
We find all three-dimensional Einstein-Weyl spaces with the vanishing scalar curvature.

Three-dimensional Lorentzian Einstein metrics have constant curvature. In order to construct nontrivial gravitational models in three dimensions, one should therefore look at conformal geometries involving a non-metric connection. Einstein-Weyl geometries appear quite naturally in this context.

Let $\mathcal{W}$ be a three-dimensional Weyl space i.e. a manifold with with a torsion-free connection $D$ and a conformal metric $[h]$ such that null geodesics of $[h]$ are also geodesics for $D$. This condition is equivalent to $D_{i} h_{j k}=\omega_{i} h_{j k}$ for some one form $\omega$. Here $h_{j k}$ is a representative metric in the conformal class, and the indices $i, j, k, \ldots$ go from 1 to 3 . If we change this representative by $h \longrightarrow \phi^{2} h$, then $\omega \longrightarrow \omega+2 \mathrm{~d} \ln \phi$. The one-form $\omega$ 'measures' the difference between $D$ and the Levi-Civita connection $\nabla$ of $h$. A tensor object $T$ which transforms as $T \longrightarrow \phi^{m} T$ when $h_{i j} \longrightarrow \phi^{2} h_{i j}$ is said to be conformally invariant of weight $m$. The formula for a covariant weighted derivative of a one-form of weight $m$ is

$$
D_{i} V_{j}=\nabla_{i} V_{j}+\frac{1}{2}\left((1-m) \omega_{i} V_{j}+\omega_{j} V_{i}-h_{i j} \omega_{k} V^{k}\right) .
$$

The Ricci tensor $W_{i j}$ is related to the Ricci tensor $R_{i j}$ and of $\nabla$ by

$$
W_{i j}=R_{i j}+\nabla_{i} \omega_{j}-\frac{1}{2} \nabla_{j} \omega_{i}+\frac{1}{4} \omega_{i} \omega_{j}+h_{i j}\left(-\frac{1}{4} \omega_{k} \omega^{k}+\frac{1}{2} \nabla_{k} \omega^{k}\right) .
$$

The conformally invariant Einstein-Weyl $(\mathrm{EW})$ condition on $(\mathcal{W}, h, \omega)$ is $W_{(i j)}=W h_{i j} / 3$, or in terms of the Riemannian data:

$$
\chi_{i j}:=R_{i j}+\frac{1}{2} \nabla_{(i} \omega_{j)}+\frac{1}{4} \omega_{i} \omega_{j}-\frac{1}{3}\left(r+\frac{1}{2} \nabla^{k} \omega_{k}+\frac{1}{4} \omega^{k} \omega_{k}\right) h_{i j}=0,
$$

where $\chi_{i j}$ is the trace-free part of the Ricci tensor of the Weyl connection, and $r=h^{i j} R_{i j}$. Weyl spaces which satisfy (2) will be called Einstein-Weyl spaces. The EW equations can be regarded as an integrable system. This is because both the twistor theory [5] and the Lax representation [4] exist. One should therefore be able to construct large families of explicit solutions.

In this paper we shall find explicitly all EW spaces with vanishing scalar curvature $W=h^{i j} W_{i j}$. We shall first establish the following result:

Lemma 1 If the scalar curvature of the Weyl connection vanishes on and $E W$ space $(\mathcal{W},[h], D)$, then the Faraday two form $F_{i j}:=\nabla_{[i} \omega_{j]}$ is null. 
Proof. The Bianchi identities for the curvature of the Weyl connection written in terms of the Levi-Civita connection and $\omega$ are [6]

$$
\nabla^{i} F_{i j}+\frac{1}{2} \omega^{i} F_{i j}+\frac{1}{3}\left(\nabla_{j} W+\omega_{j} W\right)=0 .
$$

Assume $W=0$ (this is a well defined condition as $W$ is conformally invariant of weight -2 ). Contracting (3) with $\nabla^{j}$, and using $\nabla^{j} \nabla^{i} F_{i j}=0$ yields

$$
0=\left(\nabla^{j} \omega^{i}\right) F_{i j}+\omega^{i} \nabla^{j} F_{i j}=F^{i j} F_{i j}-\frac{1}{2} \omega^{i} \omega^{j} F_{i j},
$$

so $F$ is null.

We conclude that there are no non-trivial scalar-flat EW spaces in the Euclidean signature [1]. Nontrivial solutions can be found in the indefinite signature:

Proposition 2 Let $(h, \omega)$ be an Einstein-Weyl structure with vanishing scalar curvature. Then either $(h, \omega)$ is flat, or the signature is $(++-)$ and there exist local coordinates $x^{i}=(y, x, t)$ such that $\omega=y \mathrm{~d} t$, and $h$ is given by one of two solutions:

$$
\begin{gathered}
h_{1}=\mathrm{d} y^{2}+2 \mathrm{~d} x \mathrm{~d} t+\left(x\left[R(t)-\frac{y}{2}\right]+\frac{1}{48} y^{4}+\frac{1}{12} R(t) y^{3}+S(t) y\right) \mathrm{d} t^{2}, \\
h_{2}=\mathrm{d} y^{2}+2 \mathrm{~d} x \mathrm{~d} t-\frac{4 x}{y} \mathrm{~d} y \mathrm{~d} t+\left(\frac{x^{2}}{y^{2}}+\frac{x y}{2}+\frac{1}{8} y^{4}+R(t) y^{2}+S(t) y\right) \mathrm{d} t^{2},
\end{gathered}
$$

where $R(t)$ and $S(t)$ are arbitrary functions with continuous second derivatives.

Proof. Lemma 1 implies that $F_{i j}=\nabla_{[i} \omega_{j]}$ is a closed null two-form. The conformal freedom together with the Darboux theorem imply the existence of coordinates such that $\omega_{i}=y \nabla_{i} t$. Therefore $\omega \wedge \mathrm{d} \omega=$ 0 , and the nullity of $F$ gives $* F \wedge \omega=0$. We can rewrite Bianchi identity (3) as

$$
2(\mathrm{~d} * F)+\omega \wedge * F=0,
$$

and deduce $\mathrm{d} * F=0$. Therefore $\nabla^{i} F_{i j}=0$, and $\varepsilon_{i}{ }^{j k} F_{j k}=f(t) \nabla_{i} t$. Redefining $y, t$ we can set $f(t)=1$. The most general metric consistent with $\mathrm{d} t=* \mathrm{~d} y \wedge \mathrm{d} t$ is

$$
h=\mathrm{d} y^{2}+2(\hat{E} \mathrm{~d} s+\hat{F} \mathrm{~d} y+\hat{G} \mathrm{~d} t) \mathrm{d} t,
$$

where $\hat{E}, \hat{F}$, and $\hat{G}$ are functions of $(s, y, t)$. Put $\hat{E}=\partial x / \partial s$ and define $G=\left(\hat{G}-x_{t}\right) / 2, F=\hat{F}-x_{y}$, so that

$$
h=\mathrm{d} y^{2}+2 \mathrm{~d} x \mathrm{~d} t+2 F \mathrm{~d} y \mathrm{~d} t+G \mathrm{~d} t^{2}, \quad \omega=y \mathrm{~d} t .
$$

The freedom $x \rightarrow x+P(y, t)$ implies that $F(x, y, t)$, and $G(x, y, t)$ are defined up to addition of derivatives of $P(y, t)$. Furthermore the conformal scale is only fixed up to arbitrary functions of $t$, $h \mapsto \tilde{h}=\Omega h$. This leads to to the redefinitions $(x, y, t) \rightarrow(\tilde{x}, \tilde{y}, \tilde{t})$, given by

$$
\tilde{t}=T(t), \quad \tilde{y}=\frac{y}{T_{t}}-2 \frac{T_{t t}}{T_{t}}, \quad \tilde{x}=\frac{x}{T_{t}^{3}}+P(y, t), \quad \Omega=T_{t}^{2} .
$$

These transformations will latter be used to simplify $F$ and $G$. Now impose the EW equations: Equation $\chi_{12}=0$ implies $F_{x x}=0$, and we can choose $P(y, t)$ such that $F=x l(y, t)$. Now $\chi_{12}=\chi_{22}=$ 
0 . Equations $\chi_{11}=0, \chi_{23}=0$ are equivalent and imply $a_{y}=G_{x x}$. Take $G=x^{2} l_{y} / 2+x m(y, t)+n(y, t)$. The vanishing of the scalar curvature

$$
W=r+2 \nabla^{k} \omega_{k}-\frac{1}{2} \omega^{k} \omega_{k}=\left(3 l^{2}-6 l_{y}\right) / 2
$$

gives $l^{2}=2 l_{y}$, therefore $l(y, t)=0$ (case $\left.\mathbf{1}\right)$, or $l(y, t)=-2 /(y+c(t))($ case $\mathbf{2})$.

- In case $1 \chi_{31}=0$ implies $m(y, t)=R(t)-y / 2$. Finally $\chi_{33}=0$ yields $n(y, t)=y^{4} / 48+$ $\left.R(t) y^{3} / 12+S(t) y+Z(t)\right)$. Redefining $x$, we set $Z(t)=0$, and the metric is given by (画.

- In case 2 the conformal freedom $(\overline{7})$ can be used to eliminate $c(t)$. This is achieved by setting $T_{t t}=c(t)$, and redefining $m(y, t)$, and $n(y, t)$. Now $\chi_{31}=0$ implies $m(y, t)=y / 2+P(t)$, and $\chi_{33}=0$ gives $n(y, t)=y^{4} / 8+y^{3} P(t) / 4+R(t) y^{2}+S(t) y$. In fact we can get rid of $P(t)$ : Replace $x$ by $x-y^{2} w(t) / 2$, and redefine $R(t)$ to obtain (5).

The next proposition shows that there doesn't exist a combination of coordinate and conformal transformations which maps $\left(h_{1}, \omega\right)$ to $\left(h_{2}, \omega\right)$. Cases $\mathbf{1}$ and $\mathbf{2}$ are essentially distinct and can be invariantly characterized:

Proposition 3 Let $(h, \omega)$ be a non-flat $E W$ structure with a vanishing scalar curvature, and let $F$ be a corresponding Faraday two form. If $* F$ is parallel with respect to a weighted Weyl connection, then $(h, \omega)$ is locally given by (4). Otherwise it is locally given by (5).

Proof. In both cases $\mathbf{1}$ and $\mathbf{2} * F=\varepsilon_{i}{ }^{j k} F_{j k} \mathrm{~d} x^{i}=\mathrm{d} t$. First notice that vanishing of $D(f \mathrm{~d} t)$ for some $f$ is invariant condition; in a conformal scale defined by $f$ the one-form $* F$ is parallel. Here we treat $f \mathrm{~d} t$ as a weighted object. In case 1 we find that with $m=3 / 2$ we have $D(f \mathrm{~d} t)=0$ if $f=f(t)$, and $f_{t}+f R / 2=0$. In case $\mathbf{2} D(f \mathrm{~d} t)$ doesn't vanish for any $f$. For example $(D(f \mathrm{~d} t))_{13}=-f / y$.

Using the formula for a weighted derivative of a vector of weight $m$

$$
D_{i} V^{j}=\nabla_{i} V^{j}-\frac{1}{2} \delta_{i}^{j} \omega_{k} V^{k}-\frac{m+1}{2} \omega_{i} V^{j}+\frac{1}{2} \omega^{j} V_{i}
$$

we deduce that the EW structure (41) (case 1) admits a covariantly constant vector

$$
V=\exp \left(-\frac{1}{2} \int^{t} R(t) \mathrm{d} t\right) \frac{\partial}{\partial x}
$$

with weight $-1 / 2$. Therefore $\left(h_{1}, \omega\right)$ belongs to the conformal class of the dKP Einstein-Weyl spaces 沟.

It is natural to ask if other special classes of Einstein-Weyl spaces include Lorentzian scalar-flat examples. Along these lines, we have the following observation.

Proposition 4 Let $R$ be an arbitrary function of one variable. Then the Weyl structure

$$
h=4 \frac{(z+R(v))^{2}}{(1+v w)^{2}} \mathrm{~d} v \mathrm{~d} w+\mathrm{d} z^{2}, \quad \omega=\frac{4}{z+R(v)} \mathrm{d} z
$$

is scalar-flat, and $u=2 \log (2(z+R(v)) /(1+v w))$ is a solution of the Lorentzian $S U(\infty)$ Toda equation $4 u_{v w}+\left(e^{u}\right)_{z z}=0$.

Proof. These are straightforward verifications, using the fact that the scalar curvature of the Weyl structure $\left(h=e^{u} \mathrm{~d} v \mathrm{~d} w+\mathrm{d} z^{2}, \omega=2 u_{z} \mathrm{~d} z\right)$ is $\frac{1}{2} u_{z z}+\frac{1}{4} u_{z}^{2}$ : these spaces are Lorentzian analogues of the hyperCR-Toda Einstein-Weyl spaces [2], see also [7]. 


\section{References}

[1] Calderbank, D.M.J. The Farraday 2-form in Einstein-Weyl geometry, Warwick Preprint $23 / 1998$.

[2] Calderbank, D.M.J. \& Tod, K.P. Einstein metrics, hypercomplex structures and the Toda field Diff. Geom. Appl. 15, 199-208.

[3] Cartan, E. (1943) Sur une classe d'espaces de Weyl, Ann. Sci. Ecole Norm. Supp. 60, 1-16.

[4] Dunajski, M., Mason, L.J., \& Tod, K.P. (2001) Einstein-Weyl geometry, the dKP equation and twistor theory, J. Geom. Phys. 37, 63-92.

[5] Hitchin, N. (1982) Complex manifolds and Einstein's equations, in Twistor Geometry and Non-Linear systems, LNM 970, ed. Doebner, H.D. \& Palev, T.D.

[6] Pedersen, H. \& Tod, K.P. (1993) Three-Dimensional Einstein-Weyl Geometry, Adv. in Math. 97 74-109.

[7] Ward, R. S. (1990), Einstein-Weyl spaces and SU( $\infty)$ Toda fields, Class. Quantum Grav. 7 (1990) L95-L98. 